Annuaire suisse de politique de développement

23-1 | 2004

Faits et statistiques 2004

\title{
13. Législation du commerce de l'art en Suisse
}

\section{Catherine Schümperli Younossian}

\section{OpenEdition}

\section{Journals}

Édition électronique

URL : http://journals.openedition.org/aspd/483

DOI : 10.4000/aspd.483

ISSN : 1663-9669

\section{Éditeur}

Institut de hautes études internationales et du développement

\section{Édition imprimée}

Date de publication : 1 avril 2004

Pagination : 225-234

ISSN : 1660-5934

\section{Référence électronique}

Catherine Schümperli Younossian, «13. Législation du commerce de l'art en Suisse », Annuaire suisse de politique de développement [En ligne], 23-1 | 2004, mis en ligne le 16 mars 2010, consulté le 08 septembre 2020. URL : http://journals.openedition.org/aspd/483 ; DOI : https://doi.org/10.4000/aspd. 483 


\section{Législation du commerce de l'art en Suisse*}

a SUISSE occupe une place importante sur le marché de l'art international, arrivant au quatrième rang des nations actives sur ce marché. Mais la Suisse souffre aussi de la réputation d'être une "plaque tournante» pour le commerce illicite de biens culturels. Cette réputation était notamment entretenue par le fait que la Suisse n'avait pas de législation fédérale en la matière, ni ratifié les principales conventions internationales traitant du commerce de biens culturels. Il aura fallu près de quinze ans pour concilier les positions antagonistes des principaux acteurs (marchands et collectionneurs d'art d'une part, et milieux culturels et organisations de développement d'autre part) et pour qu'un projet de loi puisse être proposé au Parlement suisse. Ainsi, les Chambres fédérales ont adopté en juin 2003 la Loi fédérale sur le transfert des biens culturels (LTBC) et accepté de ratifier la convention internationale de l'UNESCO de 1970. La nécessité de redéfinir les règles du jeu en matière de commerce de biens culturels en Suisse a été notamment portée par les milieux culturels et les organisations de développement, avec le souci de préserver le patrimoine culturel des pays en développement, spécifiquement lorsque les biens archéologiques et ethnographiques sont concernés.

Le commerce de l'art est un marché singulier de par les objets qui sont échangés et par le trafic illicite qui le caractérise. Dans son message ${ }^{1}$, le Conseil fédéral relève la particularité des biens culturels: «Ils sont en effet les sources de l'identité individuelle et collective, les témoins uniques et irremplaçables de la culture et de l'histoire. Ils façonnent l'image que l'individu et la société se font d'eux-mêmes et favorisent la cohésion sociale. $\aleph^{2}$ De même, le message précise que «la demande accrue en objets archéologiques et ethnologiques a fait que le commerce illégal en est devenu très lucratif et qu'il a été repris en plusieurs endroits par la criminalité organisée». Et de préciser «qu'il s'accompagne de vols, du pillage et de la destruction de sites archéologiques [...]. Si tous les pays en pâtissent, ce sont avant tout les régions culturellement riches, mais dont les ressources ou les structures étatiques ne suffisent pas à assurer une protection efficace du patrimoine, qui sont les plus touchées $»^{3}$.

\section{$\square$ Quelques données chiffrées}

Durant ces dernières décennies, le commerce international de biens culturels a pris une importance grandissante au niveau mondial. Une étude publiée en $2002^{4}$ nous donne de précieux éléments quant au commerce international de l'art dans son ensemble, ainsi que la place relative qu'y occupent les différents

* Par Catherine Schümperli Younossian, chargée de recherche à l'iuéd.

1 Conseil fédéral, Message relatif à la Convention de l'UNESCO de 1970 et à la Loi fédérale sur le transfert international des biens culturels (LTBC) du 21 novembre 2001 (FF 2002 505).

Ibid., p. 509.

Ibid.

4 S. Guex, «Le marché suisse de l'art 1886-2000. Un survol chiffré», in Guex S. et Lafontant Vallotton C. (dir), Le marché suisse de l'art (XIXe et XX siècles), Traverse. Revue d'histoire, ${ }^{\circ}$ 1, 2002, pp. 29-62. 
pays. Son auteur relève que «considéré globalement, le marché mondial connaît une croissance soutenue entre 1970 et le milieu des années 1980 puisque les importations comme les exportations passent de près de 500 millions de dollars à un niveau situé entre 3 et 4 milliards de dollars en 1985. Durant la seconde moitié des années 1980, le marché explose: à son sommet, en 1990, les importations comme les exportations frôlent 14 milliards de dollars. Dès 1991, on assiste à un brutal affaissement, les échanges mondiaux atteignent à peine la moitié niveau de 1990. Depuis lors, le marché semble péniblement remonter la pente». Les fluctuations sur le marché de l'art s'expliquent notamment par la croissance sans précédent qu'ont connue les maisons de ventes aux enchères de 1985 à 1989, correspondant à l'explosion de la demande sur le marché international et à l'envolée des prix devenue exponentielle à partir de 1987. Le krach boursier d'octobre 1987, le minikrach d'octobre 1989, la guerre du Golfe de 1990, le marasme économique, et plus directement lié au marché de l'art le retrait des acheteurs japonais, ont contribué à faire baisser la demande d'œuvres d'art. Au cours de la saison 1990-1991, le chiffre d'affaires de Sotheby's a diminué de $59 \%$ par rapport à celui de l'année précédente, celui de Christie's de $49 \%$ et celui de l'Hôtel Druot de $43 \%$.

Concernant la place qu'occupent les différents pays, l'étude de Sébastien Guex ${ }^{6}$ relève que les Etats-Unis dominent le marché mondial de l'art avec une part de l'ordre d'un tiers, devançant nettement le Royaume-Uni qui atteint un quart environ. Elle estime enfin que «loin derrière vient un trio probablement emmené par la Suisse, dont la fraction, sans doute sous-estimée en raison de son importance dans le commerce souterrain et/ou illégal, tourne autour de $9 \%$, suivie de près par l'Allemagne avec $8 \%$ environ et la France $(5 \%) »^{7}$. Ces données chiffrées ne prennent évidemment pas en compte le trafic illicite, dont le revenu annuel est estimé à 6 milliards de dollars ${ }^{8}$.

\section{$\square$ La Suisse et le marché de l'art}

Selon la Statistique annuelle du commerce extérieur de la Suisse, les importations de biens culturels ont atteint plus de 1,1 milliard de francs en l'an 2002 et les exportations se montent à environ 1,3 milliard pour la même année.

Tableau 38: Importation et exportation suisses d'œuvres d'art et d'antiquité, 1999-2002 (en millions de francs)

\begin{tabular}{lcccc}
\hline & $\mathbf{1 9 9 9}$ & $\mathbf{2 0 0 0}$ & $\mathbf{2 0 0 1}$ & $\mathbf{2 0 0 2}$ \\
\hline Importation & 1002 & 1359 & 1594 & 1106 \\
\hline Exportation & 955 & 1435 & 1101 & 1337 \\
\hline
\end{tabular}

Source: Direction générale des douanes, Statistique du commerce extérieur de la Suisse. Rapport annuel 2002, Berne, 2003.

Les avis sont partagés sur les raisons qui ont contribué à ce que la Suisse devienne une des premières places du marché international de l'art. Comme le souligne le message du Conseil fédéral, «elle le doit en premier lieu à l'expérience et à la compétence de ses marchands d'art, lesquels savent exploiter les

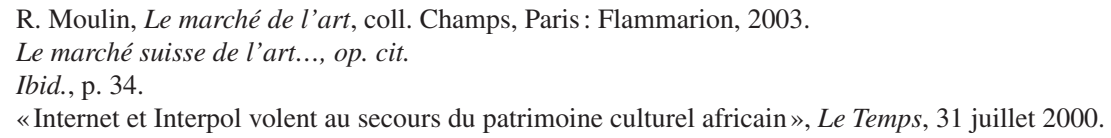


conditions attrayantes qui résultent de leurs bons contacts internationaux, de la position géographique centrale du pays et du développement de ses infrastructures». Mais le message relève aussi que «la Suisse a la réputation d'être une plaque tournante du trafic illicite des biens culturels » ${ }^{9}$. Il est vrai que ces dernières années, de nombreuses affaires de vols et de recels d'œuvre d'art ont fait la une de la presse suisse et internationale, mettant ainsi la Suisse sous le feu de la critique. Un autre élément qui tend à démontrer le rôle de la Suisse est le nombre croissant de demandes d'entraide judiciaire concernant les biens culturels volés ou illicitement exportés ${ }^{10}$.

De fait, la Suisse a bénéficié, jusqu'à la toute récente adoption de la Loi fédérale sur le transfert international de biens culturels (LTBC) présentée dans cet article, d'un contexte juridique peu contraignant pour le commerce de l'art. En effet, contrairement aux autres nations importantes dans ce commerce, la Suisse ne connaissait pas, à l'échelon fédéral, de dispositions légales spécifiques et applicables en matière de négoce de biens culturels, domaine de la compétence des cantons selon la Constitution suisse. De même, elle n'était pas rattachée aux instruments internationaux visant à réglementer le transfert de biens culturels (voir ci-dessous). L'existence de ports francs (Genève, Zurich et Bâle) permettait l'importation, l'exportation et l'entreposage de biens culturels sans grande transparence (aucun registre des biens entreposés n'était, par exemple, obligatoire). Ces lacunes juridiques ont permis d'alimenter la mauvaise réputation de la Suisse ${ }^{11}$.

\section{$\square$ Conventions internationales relatives au commerce de biens culturels}

$\mathrm{Au}$ niveau international, il existe deux conventions traitant du commerce de biens culturels: la Convention de l'UNESCO du 14 novembre 1970 concernant les mesures à prendre pour empêcher l'importation, l'exportation et le transfert de propriété illicites des biens culturels ${ }^{12}$ et la Convention d'Unidroit ${ }^{13} d u$ 24 juin 1995 sur les biens culturels volés et illicitement exportés.

\section{$\square$ La convention de l'UNESCO de 1970}

La convention exige la restitution de matériel artistique volé et exporté illégalement. Elle n'a pas d'effet rétroactif et englobe uniquement les objets provenant de musées et d'institutions analogues. Les Etats signataires s'engagent en outre à contrôler l'exportation et l'importation de biens culturels, à collaborer à l'établissement d'inventaires et à s'informer du traitement de ces objets; ils s'engagent également à se soutenir mutuellement lors de découverte de transactions illégales. A la fin 2003, 102 Etats y ont adhéré, parmi lesquels de nombreux Etats du Sud, ainsi que neuf membres de l'Union européenne (Danemark, Espagne, Finlande, France, Grèce, Italie, Portugal, Royaume-Uni, Suède), l'Australie, les Etats-Unis et le Canada. Etant d'application indirecte (non selfexecuting), les dispositions de la convention doivent d'abord être formellement intégrées dans la législation de chaque Etat contractant. Un certain nombre de

\footnotetext{
Message relatif à la Convention de l'UNESCO de 1970..., op. cit., p. 515.

10 Ibid.

11 «Visite guidée dans les ports francs de Genève », Le Temps, 24 novembre 2003.

12 Pour toute information sur la convention : <www.unesco.org/culture/laws/1970/html_fr/page1.shtml $>$.

13 Institut international pour l'unification du droit privé (Unidroit) : <www.unidroit.org $>$.
} 
critiques ont été émises à l'encontre de la convention. Sa formulation, très générale, laisse une importante liberté dans sa transposition dans le droit national, en particulier pour la définition des biens culturels. Certains reprochent au texte des préoccupations trop unilatérales; d'où son peu de succès auprès des Etats «importateurs» de biens culturels. De plus, la convention se caractérise par un champ d'application extrêmement limité, puisqu'elle ne traite que de la restitution des biens culturels volés dans les musées ou les institutions analogues. Consciente de ces limites, l'UNESCO avait demandé à l'Institut international pour l'unification du droit privé (Unidroit) d'étudier le champ privé, ce qui a donné naissance à la Convention d'Unidroit du 24 juin 1995 sur les biens culturels volés et illicitement exportés.

\section{La convention d'Unidroit}

Le commerce de l'art étant principalement d'ordre privé (contrats de vente, transferts de propriété à un acquéreur de bonne foi, etc.), il devenait indispensable d'aborder ce problème sur le terrain du droit privé. L'objectif est d'éviter que les milieux intéressés tirent parti de la diversité des lois en vigueur dans les différents pays, tout en relayant efficacement les déclarations de principe qui fondent la convention de l'UNESCO. La convention d'Unidroit permet de lutter contre les pratiques illicites dans le commerce international de biens culturels. Parallèlement, elle met à la disposition de tout propriétaire légitime, qu'il s'agisse d'un Etat, d'un musée ou d'un collectionneur, un outil juridique lui donnant les moyens de faire valoir ses droits devant un tribunal ordinaire pour retrouver des biens culturels volés ou illicitement exportés. Par conséquent, si un possesseur ou un Etat étrangers faisaient valoir des droits de restitution, il reviendrait aux tribunaux et aux juges suisses de décider de la légitimité de ces droits.

A la différence de la convention de l'UNESCO, la convention d'Unidroit est d'application directe (self-executing), c'est-à-dire qu'il n'est pas nécessaire d'intégrer ses dispositions dans la législation nationale des Etats contractants. Ainsi sont énoncées dans un acte d'importance générale les conditions garantissant le retour ou la restitution des biens culturels volés ou exportés illicitement et réglant la procédure permettant de faire valoir des droits de restitution à l'échelon international. Deux points méritent d'être relevés. Premièrement, la convention n'a pas d'effet rétroactif. Elle ne s'applique pas aux biens culturels qui ont été volés ou exportés illicitement avant son entrée en vigueur. Deuxièmement, elle prévoit le paiement d'une indemnité équitable à tout possesseur de bonne foi d'un bien culturel volé ou illicitement exporté lorsqu'il a restitué le bien en question.

En juin 1996, le Conseil fédéral a constitué un groupe de travail chargé d'éclaircir un certain nombre de questions juridiques soulevées par les deux conventions. Le groupe de travail interdépartemental a rédigé un rapport, Transfert international de biens culturels ${ }^{14}$. Dans ses conclusions, le rapport souligne «qu'Unidroit est un instrument permettant de faire face efficacement aux problèmes qui se posent en matière de transfert international de biens culturels.

14 Office fédéral de la culture, Transfert international de biens culturels, rapport du groupe de travail,


3000 Berne. Sur Internet: <www.kultur-schweiz.admin.ch/arkgt/kgt/index_f.htm>, sous «Nos publications ». 
Une ratification de la convention de l'UNESCO se justifie en particulier en ceci que cette convention crée un cadre multilatéral pour la coopération internationale en matière de protection internationale de biens culturels ». Et de rappeler qu'aucun obstacle de droit constitutionnel ni de droit privé ne s'oppose à la ratification des deux conventions. L'option de la ratification des deux conventions ainsi que la rédaction d'une loi d'application en droit suisse sont retenues par le groupe de travail.

Le Conseil fédéral n'a toutefois pas suivi les recommandations du groupe d'experts puisque le message soumis aux Chambres concerne uniquement la ratification de la convention de l'UNESCO de 1970, ainsi qu'une loi fédérale d'application. Quant à la convention d'Unidroit, le Conseil fédéral considère que cette décision est prématurée. Il a toutefois chargé le DFI «de poursuivre l'examen de l'évolution internationale, et le moment venu, de faire des propositions au Conseil fédéral $»^{15}$.

\section{$\square$ Un long processus législatif}

Il aura fallu environ treize ans, depuis la constitution d'un premier groupe de travail chargé d'étudier la question de l'exportation des biens culturels suisses et de l'importation de biens culturels étrangers, en 1990, jusqu'à l'approbation par les Chambres d'une loi régissant cette matière en juin 2003. C'était manifestement le temps nécessaire pour concilier deux clans antagonistes, d'une part, les partisans d'une double ratification des deux conventions internationales (UNESCO et Unidroit) et d'une sévère législation suisse, constitués principalement par les milieux culturels et les ONG de développement, et, d'autre part, certains milieux de commerçants et collectionneurs qui considéraient qu'une législation imposerait de telles contraintes que leurs activités en seraient entravées.

De nombreuses procédures de consultation et auditions publiques ${ }^{16}$ auront été nécessaires pour que l'Office fédéral de la culture arrive à proposer un projet de loi dont l'existence même ne soit pas contestée par les milieux concernés.

L'opposition des milieux de l'art au projet de loi était très forte et s'est concrétisée par une initiative parlementaire (initiative Fischer ${ }^{17}$ ) présentée à titre de contre-projet. L'initiative parlementaire Fischer proposait un affaiblissement de la LTBC (notamment relatif au délai de restitution, dix ans seulement, et à un assouplissement des clarifications exigées sur l'origine des biens culturels importés). A l'inverse, 21 ONG suisses se sont regroupées au sein d'une plateforme, coordonnée par la Déclaration de Berne ${ }^{18}$, invitant les parlementaires suisses à voter en faveur du projet, mais rappelant que la signature de la convention d'Unidroit constituait une future étape importante pour un échange

15 Département fédéral de l'intérieur, Rapport du groupe de travail. Transfert international de biens culturels, communiqué de presse, 26 août 1998.

16 Procédure de consultation concernant la convention de l'UNESCO de 1970 (1993), Procédure de consultation concernant la convention d'Unidroit (1996), Groupe de travail interdépartemental (19961998), Auditions publiques (1999). Pour plus de détails, se rapporter au Message relatif à la convention de l'UNESCO de 1970..., op. cit.

17 Initiative parlementaire «Promulgation d'une loi fédérale sur le transfert de biens culturels», $\mathrm{n}^{\circ}$ 01.450. L'initiative a été présentée lors de la session de printemps 2003 du Conseil national, puis retirée à l'issue des débats de la Chambre basse.

18 Site de la Déclaration de Berne : <www.ladb.ch>. 
équitable des biens culturels ${ }^{19}$. La Commission suisse pour l'UNESCO ${ }^{20}$ a été le fer de lance des milieux culturels pour promouvoir la ratification des deux conventions et exiger une loi d'application.

\section{$\square$ Message du Conseil fédéral}

Par le message du 21 novembre 2001 présenté ci-dessous, le Conseil fédéral a ainsi proposé aux Chambres fédérales de ratifier la convention de l'UNESCO de 1970 concernant les mesures à prendre pour interdire et empêcher l'importation, l'exportation et le transfert de propriété illicites des biens culturels et a soumis un projet de loi fédérale sur le transfert international de biens culturels (LTBC).

En proposant au Parlement la ratification de la convention de l'UNESCO et sa loi d'application, le Conseil fédéral a notamment fait valoir l'importance du commerce de l'art, mais en souhaitant clairement «permettre de barrer la route au commerce illégal - et moralement répréhensible - desdits biens en Suisse. Les abus seront combattus de manière ciblée $»^{21}$.

\section{Loi fédérale sur le transfert international de biens culturels (LTBC)}

La LTBC poursuit deux objectifs majeurs: d'une part, la protection du patrimoine culturel suisse (en invitant la Confédération et les cantons à dresser un inventaire des biens culturels et en édictant des dispositions réglant l'exportation des biens culturels d'importance significative appartenant à la Confédération); d'autre part, la mise en place d'un cadre juridique visant à réglementer le commerce international de l'art.

Ainsi, la LTBC met en œuvre les obligations de la convention de l'UNESCO pour lesquelles la Confédération est compétente, en répondant notamment aux objectifs suivants :

$\checkmark$ créer des bases légales permettant de lutter contre les abus en matière de transfert de biens culturels;

$\checkmark$ renforcer la coopération internationale dans le domaine du transfert de biens culturels;

$\checkmark$ adapter la législation aux normes internationales minimales;

- favoriser l'ouverture et l'équité des échanges internationaux de biens culturels.

La loi comprend des dispositions régissant l'importation, l'exportation et le commerce de biens culturels. Elle prévoit des mesures qui améliorent la protection du patrimoine culturel de la Suisse et des autres Etats parties à la convention de l'UNESCO; elle vise aussi à favoriser les échanges culturels. Sont présentés ci-dessous quelques aspects importants de la loi:

$\checkmark$ Concernant l'importation en Suisse de biens culturels, la loi prévoit deux mesures (art. 7 et 8 LTBC) : premièrement, à la demande d'un Etat partie à la convention de l'UNESCO, la Suisse conclut un accord bilatéral qui règle les importations de biens culturels d'importance significative pour le patrimoine culturel de l'Etat concerné; deuxièmement, le Conseil fédéral peut prendre

19 Prise de position des ONG au sujet de la Loi fédérale sur le transfert international des biens culturels (30 avril 2002), disponible sur le site de la Déclaration de Berne : <www.ladb.ch>.

20 Site de la Commission suisse pour l'UNESCO: <www.unesco.ch>.

21 Message relatif à la convention de l'UNESCO de 1970..., op. cit., p. 507. 
des mesures temporaires quant à l'importation et l'exportation de biens culturels provenant de territoires victimes d'événements extraordinaires (guerres, catastrophes naturelles).

ـ En matière d'exportation de biens culturels, sont concernés uniquement les biens appartenant à la Confédération (pour le reste des biens culturels, ce sont les cantons qui sont compétents). Un inventaire fédéral des biens appartenant à la Confédération sera établi, avec pour conséquence que les biens inscrits seront souscrits au commerce et qu'il est interdit de les exporter définitivement hors de Suisse. Les organes douaniers doivent contrôler l'exportation de biens culturels hors de Suisse (art. 3 à 6 et 19 LTBC).

$\checkmark$ Au chapitre du transfert de biens culturels, la loi énonce les règles auxquelles sont soumises les institutions fédérales lorsqu'elles acquièrent un bien culturel. La loi édicte un certain nombre de règles concernant le commerce d'objets d'art et les ventes aux enchères. Les commerçants se voient ainsi dans l'obligation d'enregistrer l'origine de biens culturels, d'informer leur clientèle des règlements concernant l'importation et l'exportation, de tenir un registre des acquisitions de biens culturels et de fournir à un service spécialisé (à créer en cas d'adoption de la LTBC) tous les renseignements nécessaires concernant l'accomplissement de ce devoir de diligence (art. 15 à 17 LTBC). Enfin, le délai de prescription acquisitive et de restitution des biens culturels volés est porté à trente ans (art. 32 LTBC). C'est précisément la question des délais qui a suscité de vifs débats aux Chambres (voir ci-dessous).

- Parmi d'autres mesures de la LTBC, citons la possibilité qu'offre la loi à la Confédération d'accorder une aide financière destinée à sauvegarder le patrimoine culturel (art. 14 LTBC). La loi comprend également des dispositions complémentaires concernant les autorités (art. 18 à 20 LTBC), l'entraide administrative et judiciaire (art. 21 à 23 LTBC) et les sanctions (art. 24 à 29 LTBC). Enfin, il est également prévu de créer un service spécialisé qui aurait notamment pour tâche de conseiller et d'assister les autorités cantonales et fédérales, de collaborer avec les autorités d'autres Etats en vue de la protection du patrimoine culturel, etc. (art. 18 LTBC).

\section{$\square$ Débat aux Chambres}

Les Chambres fédérales ont traité de l'Arrêté fédéral portant approbation de la convention de l'UNESCO de 1970 et du projet de la LTBC lors des sessions de printemps et d'été 2003. La loi, adoptée le 20 juin 2003, est très similaire au projet du Conseil fédéral, à un point près: celui de la définition d'un bien culturel. Dans le projet de loi, le Conseil fédéral avait donné une définition large de la notion de biens culturels - finalement retenue ${ }^{22}$ - et également précisé un sens strict des biens culturels (bien archéologique, bien ethnographique, bien sacré ou archives), pour lesquels un contrôle sévère était exigé. Les Chambres ont renoncé à cette double définition; cette décision élargit, de fait, le champ d'application de la loi.

Le Conseil national s'est penché sur la loi en mars 2003 et le Conseil des Etats

22 Par «biens culturels», on entend les biens qui, à titre religieux ou profane, revêtent de l'importance pour l'archéologie, la préhistoire, l'histoire, la littérature, l'art ou la science et qui font partie de l'une des catégories prévues à l'article 1 de la convention de l'UNESCO de 1970 (art. 2, al.1 LTBC). 
en juin 2003. Trois points principaux ont suscité la discussion lors du débat parlementaire ${ }^{23}$ :

- Obligation de communiquer. La commission du Conseil national avait proposé d'introduire une obligation d'annoncer aux autorités les cas lorsqu'il y a soupçon fondé quant à une importation illégale de biens culturels ou s'il existe un soupçon de négoce d'objets volés. Cette proposition a reçu le soutien des partis de gauche et des démocrates-chrétiens. A l'opposé, les radicaux, l'UDC et les libéraux se sont dits préoccupés par une disposition qui inciterait à la délation et mettrait les marchands d'art dans une situation qui pourrait ruiner les relations de confiance entre commerçants et clients. Après le vote au national, cette proposition a été biffée.

- Délai de prescription. Le Conseil fédéral prévoyait un délai de prescription de trente ans, à savoir qu'un bien acquis de bonne foi ne doit plus être obligatoirement restitué à son propriétaire après ce délai. Le délai de trente ans correspond à la réglementation européenne en la matière. Cependant, le Conseil national a accepté une proposition d'un député de l'UDC qui visait à abaisser le délai de prescription à quinze ans. Contrairement au Conseil national, le Conseil des Etats a exigé que le délai de prescription pour le retour de biens volés passe de cinq ans, comme c'est le cas actuellement, à trente ans.

- Mode d'indemnisation. Ce point concerne le mode d'indemnisation d'un acheteur de bonne foi en possession d'un bien culturel illicitement importé. La proposition d'une députée libérale visant à définir l'indemnisation sur la base de la valeur marchande, et non pas du prix d'achat, lorsqu'un bien doit être restitué à l'Etat requérant, a été acceptée par le Conseil national. A l'inverse, le Conseil des Etats a considéré que l'indemnisation fondée sur la valeur marchande du bien est incompatible avec la convention de l'UNESCO. Ce type d'indemnisation, selon le Conseil des Etats, encouragerait la spéculation et empêcherait les pays financièrement défavorisés de récupérer les biens culturels qui leur reviennent. Le Conseil des Etats a ainsi privilégié une indemnité calculée sur le prix d'achat.

Lors de l'élimination des divergences, le Conseil national s'est rallié à toutes les décisions du Conseil des Etats. La loi a été adoptée en votation finale le 20 juin $2003^{24}$ et entrera en vigueur dans le courant de l'année 2004.

Quant à la proposition du Conseil fédéral de ratifier la convention de l'UNESCO de 1970, elle a été approuvée par les deux Chambres. Les instruments de ratification ont été déposés par la conseillère fédérale Micheline Calmy-Rey le $1^{\text {er }}$ octobre 2003, à Paris, à l'occasion de la Conférence générale de l'UNESCO ${ }^{25}$. A cette occasion, la conseillère fédérale a insisté sur le fait que par cette ratification, «la Suisse apporte une contribution importante à la conservation de l'héritage culturel de l'humanité» et confirme sa détermination à «favoriser un échange équitable et ouvert».

$\square$ Pillage des biens culturels en Irak - position de la Suisse

23 Parlement fédéral, Note de synthèse 01.077. Loi sur le transfert des biens culturels, 30 juillet 2003, $<$ www.parlament.ch $>$.

24 Texte de l'acte législatif: Loi fédérale sur le transfert international des biens culturels (LTBC) du 23 juin 2003 (FF 2003 4019).

25 Office fédéral de la culture, Ratification par la Suisse de la convention de l'UNESCO de 1970, communiqué de presse, $1^{\text {er }}$ octobre 2003. 
A la mi-avril 2003 ont eu lieu les pillages du Musée archéologique de Bagdad, ainsi que de la bibliothèque nationale irakienne, qui ont ému l'opinion publique internationale et ont, peut-être, influencé le débat parlementaire en vue de l'adoption de la LTBC. Aussitôt que les dépêches faisant état de pillages ont été rendues publiques, la communauté internationale, sous l'égide de l'UNESCO, a pris des mesures pour décourager le commerce de biens culturels en provenance d'Irak. A la mi-avril, l'Office fédéral de la culture a alerté les milieux concernés sur les risques de voir des biens culturels irakiens transiter par la Suisse, recommandant instamment de ne pas acheter ni accepter aucun bien dont l'origine n'était pas établie ${ }^{26}$. Fin mai 2003, le Conseil fédéral a, par voie d'ordonnance, interdit tout commerce de biens culturels irakiens et pris des mesures destinées à faciliter la restitution des biens culturels irakiens. Le texte de l'ordonnance précise qu'il est interdit d'importer, de faire transiter ou d'exporter, de vendre, de commercialiser, de diffuser, d'acquérir et de transférer de quelque manière que ce soit des biens culturels irakiens volés depuis le 2 août 1990 dans la République d'Irak, enlevés à leur propriétaire sans sa volonté ou exportés illégalement hors d'Irak. Cette mesure s'applique également aux biens culturels provenant de fouilles illégales. L'exportation illégale d'un bien culturel est présumée lorsqu'il est démontré que ce dernier se trouvait en Irak en date du 2 août $1990^{27}$.

Pour concrétiser son engagement envers les biens culturels irakiens, le Département fédéral des affaires étrangères a participé à hauteur de 250'000 dollars à l'initiative de l'UNESCO, également soutenue par l'Italie et le Japon, qui vise la création d'une banque de données des biens culturels irakiens.

A noter également que le Conseil fédéral a approuvé, en été 2003, le Message ${ }^{28}$ concernant le deuxième Protocole du 26 mars 1999 relatif à la Convention de La Haye ${ }^{29}$ de 1954 pour la protection des biens culturels en cas de conflit armé. Elaboré dans le cadre de l'UNESCO, ce deuxième protocole intègre les évolutions du droit international humanitaire, du droit pénal international et du droit relatif à la protection du patrimoine culturel. La Suisse, Etat partie à la convention de La Haye et à son premier protocole, a joué un rôle déterminant dans l'élaboration du deuxième protocole. Sa ratification n'exige pas une adaptation de la législation suisse.

(1] Annuaire 1997, Catherine Schümperli Younossian, «Commerce, importation et exportation de biens culturels : état de la réglementation en Suisse», Analyses et positions, pp. 271-282; 1999, «Réglementation du commerce de l'art en Suisse», pp. 195-196.

26 Office fédéral de la culture, Pillage du patrimoine irakien, communiqué de presse, 16 avril 2003.

27 Ordonnance du 28 mai 2003 instituant des mesures économiques envers la République d'Irak (RO 2003 1887), modifiant l'ordonnance homonyme du 7 août 1990 (RS 946.206).

28 Message concernant le deuxième Protocole du 26 mars 1999 relatif à la Convention de La Haye de 1954 pour la protection des biens culturels en cas de conflit armé du 20 août 2003 (FF 2003 5555).

29 La Convention pour la protection des biens culturels en cas de conflit armé, adoptée à La Haye le 14 mai 1954, est un traité destiné à prévenir les dommages et les pertes irremplaçables tels que ceux qui ont été infligés aux biens culturels pendant la Seconde Guerre mondiale. Née sous le patronage de l'UNESCO, la convention de La Haye est le premier instrument consacré exclusivement à la protection des biens culturels qui soit reconnu mondialement. 


\section{SOURCES}

Conseil fédéral, Message concernant le deuxième Protocole du 26 mars 1999 relatif à la Convention de La Haye de 1954 pour la protection des biens culturels en cas de conflit armé du 20 août 2003 (FF $20035555)$.

Conseil fédéral, Message relatif à la Convention de l'UNESCO de 1970 et à la Loi fédérale sur le transfert international des biens culturels (LTBC) du 21 novembre 2001 (FF 2002 505).

Conseil national et Conseil des Etats, Bulletin officiel, session 2003, <www.parlament.ch/ab/frameset/f/ index.htm>.

Département fédéral de l'intérieur, Loi sur le transfert des biens culturels. Rapport sur les résultats de la procédure de consultation, Berne: Office fédéral de la culture, novembre 2001.

Département fédéral de l'intérieur, Rapport du groupe de travail. Transfert international de biens culturels, communiqué de presse, 26 août 1998.

Direction générale des douanes, Statistique du commerce extérieur de la Suisse. Rapport annuel 2002, Berne, 2003.

Loi fédérale sur le transfert international des biens culturels (LTBC) du 20 juin 2003, Feuille fédérale, 2003, pp. 4019 et suiv.

Office fédéral de la culture, «Commerce des biens culturels : redéfinir les règles du jeu», OFC Journal, $5 / 2002$.

Office fédéral de la culture, Pillage du patrimoine irakien, communiqué de presse, 16 avril 2003 ; Ratification par la Suisse de la Convention de l'UNESCO de 1970, communiqué de presse, $1^{\mathrm{er}}$ octobre 2003.

Office fédéral de la culture, Transfert international de biens culturels. Convention de l'UNESCO de 1970 et Convention d'Unidroit de 1995, rapport du groupe de travail, nº 304.250 f, Berne, 1998.

Parlement fédéral, Note de synthèse 01.077. Loi sur le transfert des biens culturels, 30 juillet 2003.

Guex S. et Lafontant Vallotton C. (dir), Le marché suisse de l'art (XIXe et XXe siècles), Traverse. Revue d'histoire, $\mathrm{n}^{\circ} 1,2002$.

Moulin R., Le marché de l'art, coll. Champs, Paris : Flammarion, 2003.

Le Temps, «Internet et Interpol volent au secours du patrimoine culturel africain», 31 juillet 2000; «Marché de l'art: une loi qui criminalise ou responsabilise les marchands», 28 janvier 2003; «Biens culturels et biens publics », dossier du Samedi culturel, $1^{\text {er }}$ mars 2003 ; «Visite guidée dans les ports francs de Genève», 24 novembre 2003.

Neue Zürcher Zeitung, D. Cahn et A. Gaiser, «Unidroit, Diebstahl und illegaler Export», 23. Januar 1999.

\section{SITES INTERNET}

Commission suisse pour l'UNESCO : <www.unesco.ch $>$.

Déclaration de Berne: <www.ladb.ch $>$.

Institut international pour l'unification du droit privé (Unidroit) : <www.unidroit.org $>$.

Office fédéral de la culture/Service transfert des biens culturels : <www.culture-suisse.admin.ch $>$.

Organisation des Nations unies pour l'éducation, la science et la culture (UNESCO) : <www.unesco.org $>$.

Parlement fédéral : <www.parlament.ch>. 